\title{
Identification of Drugs Blocking SARS-CoV-2 Infection using Human Pluripotent Stem Cell- derived Colonic Organoids
}

\section{Xiaohua Duan}

Weill Cornell Medicine

Yuling Han

Weill Cornell Medicine

Liuliu Yang

Weill Cornell Medicine

Benjamin E. Nilsson-Payant

Icahn School of Medicine at Mount Sinai

Pengfei Wang

Columbia University Irving Medical Center

Tuo Zhang

Weill Cornell Medicine

Jenny Xiang

Weill Cornell Medicine

Dong Xu

Weill Cornell Medicine

Xing Wang

Weill Cornell Medicine

Skyler Uhl

Icahn School of Medicine at Mount Sinai

Yaoxing Huang

Columbia University Irving Medical Center

Huanhuan Joyce Chen

University of Chicago

Hui Wang ( $\square$ huiwang@shsmu.edu.cn )

ShanghaiTech University

Benjamin tenOever ( $\boldsymbol{\nabla}$ benjamin.tenoever@mssm.edu )

Icahn School of Medicine at Mount Sinai

Robert E. Schwartz ( $\square$ res2025@med.cornell.edu )

Weill Cornell Medicine

David. D. Ho ( $\nabla$ dh2994@cumc.columbia.edu ) 
Columbia University Irving Medical Center

Todd Evans ( $\sim$ tre2003@med.cornell.edu )

Weill Cornell Medicine

Fong Cheng Pan ( $\sim$ fcp2002@med.cornell.edu )

Weill Cornell Medicine

Shuibing Chen ( $\nabla$ hc2034@med.cornell.edu )

Weill Cornell Medicine

\section{Research Article}

Keywords: SARS-CoV-2, human colonic cell types, in vitro and in vivo organoid models, high throughput drug screening

Posted Date: April 30th, 2020

DOI: https://doi.org/10.21203/rs.3.rs-24782/v1

License: (c) (1) This work is licensed under a Creative Commons Attribution 4.0 International License. Read Full License 


\section{Abstract}

The current COVID-19 pandemic is caused by SARS-coronavirus 2 (SARS-CoV-2). There are currently no therapeutic options for mitigating this disease due to lack of a vaccine and limited knowledge of SARSCoV-2 biology. As a result, there is an urgent need to create new disease models to study SARS-CoV-2 biology and to screen for therapeutics using human disease-relevant tissues. COVID-19 patients typically present with respiratory symptoms including cough, dyspnea, and respiratory distress, but nearly $25 \%$ of patients have gastrointestinal indications including anorexia, diarrhea, vomiting, and abdominal pain. Moreover, these symptoms are associated with worse COVID-19 outcomes1. Here, we report using human pluripotent stem cell-derived colonic organoids (hPSC-COs) to explore the permissiveness of colonic cell types to SARS-CoV-2 infection. Single cell RNA-seq and immunostaining showed that the putative viral entry receptor ACE2 is expressed in multiple hESC-derived colonic cell types, but highly enriched in enterocytes. Multiple cell types in the COs can be infected by a SARS-CoV-2 pseudo- entry virus, which was further validated in vivo using a humanized mouse model. We used hPSC-derived COs in a high throughput platform to screen 1280 FDA-approved drugs against viral infection. Mycophenolic acid and quinacrine dihydrochloride were found to block the infection of SARS-CoV-2 pseudo-entry virus in COs both in vitro and in vivo, and confirmed to block infection of SARS-CoV-2 virus. This study established both in vitro and in vivo organoid models to investigate infection of SARS-CoV-2 disease-relevant human colonic cell types and identified drugs that blocks SARS-CoV-2 infection, suitable for rapid clinical testing.

\section{Main Text}

Previously, we reported a chemically-defined protocol to derive COs from hPSCs2, which we modified slightly based on published studies3. In brief, HUES8 hESCs were induced with CHIR99021 (CHIR) and Activin A to generate definitive endoderm (DE) (Extended Data Fig. 1a). After 4 days of culture with CHIR +FGF4 to induce hindgut endoderm (HE), cells were treated with BMP2, epidermal growth factor (EGF), and CHIR for 3 days to promote specification of colon progenitors (CPs). Starting on day 11, CPs were treated with a colonic medium containing CHIR, LDN193189 (LDN), and EGF. After embedding these organoids in Matrigel, spheroids became pseudostratified and progressively cavitated into fully convoluted organoids (Fig. 1a). The organoids expressed CDX2, Villin and SATB2, confirming colonic identity (Fig. 1b). Immunocytochemistry confirmed that COs contain cell types found in normal colon, including keratin 20 (KRT20)+ epithelial cells, mucin 2 (MUC2)+ goblet cells, EPH receptor B2 (EPHB2)+ transit-amplifying (TA) cells, and chromogranin A (CHGA)+ neuroendocrine (NE) cells (Fig. 1c).

Single cell RNA-seq was used to examine global transcript profiles at single cell resolution (Extended Data Fig. 1b). Consistent with the immunostaining results, most cells express $C D X 2$ and VIL 1 (Extended Data Fig. 1c). Five cell clusters were identified including KRT20+ epithelial cells, $M U C 2+$ goblet cells, $E P H B 2+$ TA cells, CHGA+ NE cells, and LGR5+ or BMI1+ stem cells (Fig. 1d-e, Extended Data Fig. 1d). We examined the expression of two factors associated with SARS-CoV-2 cell entry, the putative receptor ACE2 and the protease TMPRSS24. Both are expressed in all five cell clusters, but highly enriched in KRT20+ enterocytes (Fig. 1f-g). Two-dimensional correlation confirmed the co-expression relationship for ACE2 
and KRT20, as well as ACE2 and TMPRSS2 (Fig. 1h). Immunohistochemistry further validated the coexpression of KRT20 and ACE2 in hPSC-COs (Fig. 1i).

To model infection of hPSC-COs with SARS-CoV-2, we used a vesicular stomatitis virus (VSV) based SARS-CoV-2 pseudo-entry virus, with the backbone provided by a VSV-G pseudo-typed $\triangle$ G-luciferase virus and the SARS-CoV-2 spike protein incorporated into the surface of the viral particle (See Methods for details) 5,6. COs were fragmentized and innoculated with the SARS-CoV-2 pseudo-entry virus. 24 or $48 \mathrm{hr}$ post-infection (hpi), the cells were lysed and monitored for luciferase activity (Extended Data Fig. 2a). The organoids infected with SARS-CoV-2 pseudo-entry virus at MOI=0.01 showed a strong signal at $24 \mathrm{hpi}$ (Fig. 2a). Single cell RNA-seq was performed to examine the hPSC- derived COs at $24 \mathrm{hpi}$. The same five cell populations were identified in the COs post- infection (Fig. 2b and Extended Data Fig. 2b-d). Compared to uninfected samples, the KRT20+ enterocyte population decreased significantly (Fig. 2c). Immunostaining confirmed increased cellular apoptosis, suggesting toxicity for these cells (Extended Data Fig. 2e). In addition, the ACE2+ population was significantly depleted (Fig. 2e). The mRNAs of SARSCoV-2 pseudo-entry virus, including VSV-NS, VSV-N, and VSV-M, were detected in all five cell populations (Fig. 2f), but not in the uninfected COs (Extended Data Fig. 2f). Immunostaining further validated the expression of luciferase in ACE2,$+ V I L 1+, C D X 2+, K R T 20+$, and $M U C 2+$ cells (Fig. $\mathbf{2 g}$ ).

Humanized mice carrying hPSC-COs in vivo provide a unique platform for modeling COVID-19. In brief, hPSC-COs were transplanted under the kidney capsule of NOD-scid IL2Rgnull mice. Two weeks after transplantation, the organoid xenograft was removed and examined for cellular identities (Fig. $\mathbf{h}$ ). Consistent with in vitro culture, ACE2 can be detected in hPSC-derived KRT20+ enterocytes (Fig. 2i). SARS-CoV-2 pseudo-entry virus was inoculated locally. At $24 \mathrm{hpi}$, the xenografts were removed and analyzed by immuno-histochemistry. Luciferase was detected in the xenografts inoculated with virus, but not in MOCK-infected controls (Fig. 2j). Immunohistochemistry detected luciferase in ACE2+ and Villin+ cells, suggesting these are permissive to SARS-CoV-2 pseudo-entry virus infection in vivo (Fig. $\mathbf{2 k}$ ).

Next, we adapted hPSC-COs to a high throughput screening platform and probed the Prestwick FDAapproved drug library to identify drug candidates capable of blocking SARS-CoV-2 pseudo-virus infection. In brief, hPSC-COs were cultured in 384-well plates. After overnight incubation, organoids were treated with drugs from the library at $10 \mu \mathrm{M}$. One hour post-exposure with drugs, the organoids were innoculated with the SARS-CoV- 2 pseudo-entry virus. 24 hpi, the organoids were analyzed for luciferase activity (Fig. 3a). Drugs that decreased the luciferase activity by at least $75 \%$ were chosen as primary hit drugs (Fig. 3b). Eight drugs (Extended Data Table 1) were identified as lead hits and further tested for their capacities to decrease the luciferase signal in a dose-dependent manner (Extended Data Fig. 3). These drugs could potentially function through blocking virus entry, by decreased cell survival, or even by directly inhibiting luciferase activity. To distinguish these possibilities, the lead hit drugs were tested in comparison to hPSC-COs infected with a control VSVG-luciferase reporter virus. Four of the lead hit drugs showed specificity to SARS-CoV-2 pseudo-entry virus, including mycophenolic acid (MPA, Fig. 3c) (SARS-CoV-2:

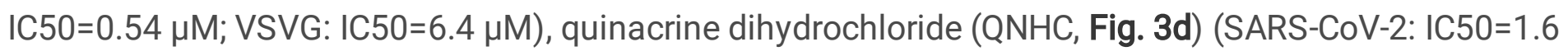

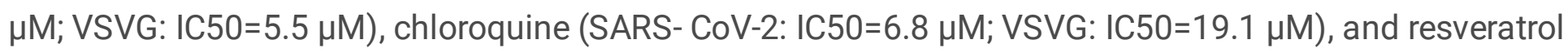




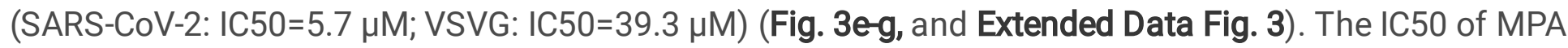
is 10 times lower and the IC50 of QNHC is 5 times lower than that of chloroquine, a drug recently authorized by the FDA for emergency use to treat COVID-19 patients7. Immunostaining confirmed few luciferase positive cells in hPSC-COs treated with $3 \mu \mathrm{M}$ MPA or $4.5 \mu \mathrm{M}$ QNHC at 24 hpi (Fig. 3h). COexplanted humanized mice were treated with $50 \mathrm{mg} / \mathrm{kg}$ MPA by IP injection, followed by local inoculation of SARS-CoV-2 pseudo-entry virus. At $24 \mathrm{hpi}$, the mice were euthanized, and xenografts were analyzed by immunostaining. Luc+ cells in the xenografts of MPA-treated mice were significantly lower than those of vehicle- treated mice (Fig. 3i-k).

Finally, hPSC-COs were infected with SARS-CoV-2 virus at MOI=0.1 or 0.01 . At $24 \mathrm{hpi}$, immunostaining detected the expression of SARS-CoV membrane protein in the infected hPSC-COs, which partially colocalized with CDX2 and KRT20 (Fig. 4a). Bulk RNA sequencing confirmed viral transcripts in the SARSCoV-2 infected hPSC-COs (MOI=0.1, Fig. 4b). The MOCK and infected hPSC-COs separated clearly into two distinct clusters in a PCA plot (Fig. 4c). Differential gene expression analysis showed striking induction of chemokine gene expression, including for IL 1A, CXCL8, CXCL6, CXCL11, and IL 1B, yet with no detectable levels of IFNH or IFN-III, which is consistent with recent reports8-10 (Fig. 4d). Ingenuity Pathway Analysis of the differential gene expression list highlighted the production of nitric oxide and reactive oxygen species, oxidative phosphorylation, as well as IL-15 production (Fig. 4e). The hPSC-COs were pre-treated with MPA or QNHC and infected with a relatively high titer of SARS-CoV-2 virus (MOI=0.1). Immunostaining confirmed the decrease of SARS-CoV-2+ cells in MPA or QNHC-treated hPSCCOs (Fig. 4f). Finally, western blotting assays confirmed the ability of MPA and QNHC to block SARS-CoV2 infection of Vero cells (Extended Data Fig. 4).

In summary, we report that hPSC-derived COs express ACE2 and TMPRS2S2 and are permissive to SARSCoV-2 infection. There is currently a lack of physiologically relevant models for COVID-19 disease that enable drug screens. Previous studies were based on clinical data or transgenic animals, for example mice that express human ACE2. However, such transgenic animals fail to fully recapitulate the cellular phenotype and host response of human cells11,12. We adapted a hPSC-derived CO platform for high throughput drug screening. Using disease-relevant normal colonic human cells, we screened 1280 FDAapproved compounds and identified MPA and QNHC, two drugs that can block the entry of SARS-CoV-2 into human cells. Strikingly, in this assay, the efficacies of MPA and QNHC for blocking viral entry are more than 5 times higher than chloroquine, a drug recently authorized by the FDA for emergency use to treat COVID-19 patients. Moreover, the MPA concentrations effective in blocking viral entry and replication are below that which is routinely used in clinical therapy13. MPA is a reversible, non-competitive inhibitor of inosine-5'-monophosphate dehydrogenase and is used widely and safely as an immunosuppressive drug (mycophenolate mofetil; CellCept) to prevent organ rejection after transplantation and for the treatment of autoimmune diseases14. MPA has been reported to block replication of human immunodeficiency virus15, dengue16, as well as Middle East respiratory syndrome coronavirus (MERSCoV)17. Several studies on MERS- CoV suggest that MPA may noncompetitively inhibit the viral papainlike protease while also altering host interferon response17,18. A recent study also predicts MPA would modulate the interaction between host protein inosine-5'-monophosphate dehydrogenase 2 (IMPDH2) 
and SARS-CoV-2 protein nonstructural protein 14 (nsp14)19. Furthermore, a clinical study of MERS-CoV suggested that the patients treated with mycophenolate mofetil has $0 \%$ mortality rate, which is significantly lower than the overall mortality rate as $37 \% 20$. QNHC (Acriquine $\AA$, Atabrine $\AA$, Atebrin $\AA$, Mepacrine $\left.{ }^{\circledR}\right)$ is an FDA-approved antimalarial drug used more recently as an anthelmintic, antiprotozoal, antineoplastic agent, and antirheumatic21. Recent studies have shown that quinacrine protects mice against Ebola virus infection in vivo 22. Both MPA and QNHC can be considered candidates for clinical trials of COVID-19 therapy.

\section{Methods}

\section{hESC maintenance and colonic lineage differentiation}

hESCs were grown and maintained on $1 \%$ Matrigel (Corning)-coated six-well plates in StemFlex medium (Gibco) at $37^{\circ} \mathrm{C}$ with $5 \% \mathrm{CO} 2$. For definitive endoderm (DE) differentiation, hESCs were cultured to achieve 80-90\% confluency, and treated with $3 \mu \mathrm{M}$ CHIR99021 (CHIR, Stem-RD) and $100 \mathrm{ng} / \mathrm{ml}$ Activin A (R\&D systems) in basal medium RPMI1640 (Cellgro) supplemented with 1X Pen-Strep (Gibco) for 1 day, and changed to the basal medium containing only $100 \mathrm{ng} / \mathrm{ml}$ Activin A the next day. To induce CDX2+ hindgut endoderm, DE were treated with $3 \mu \mathrm{M}$ CHIR99021 and $500 \mathrm{ng} / \mathrm{ml} \mathrm{FGF4} \mathrm{(Peprotech)} \mathrm{in} \mathrm{RPMI1640}$ supplemented with 1 X B27 supplement (Gibco) and 1X Pen-Strep (Gibco) for 4 days with daily changing of fresh media. Organoids began to bud out from the 2D culture during the hindgut differentiation process. The hindgut endoderm was then subjected to colonic lineage induction by treatment with 100 ng/ml BMP2 (Peprotech), $3 \mu \mathrm{M}$ CHIR99021 and 100 ng/ml hEGF (Peprotech) in Advance DMEM F12 medium supplemented with 1X B27 supplement (Gibco), 1X GlutaMax (Gibco), 10 mM HEPES (Gibco) and $1 X$ Pen-Strep (Gibco) for 3 days with daily changing of fresh medium. After colonic fate induction, the colon progenitor organoids were collected from the initial 2D cultures and embedded in a $100 \%$ Matrigel dome in a 24-well plate. Differentiation to mature colonic cell types was achieved by culturing these colon progenitor organoids in differentiation medium containing $600 \mathrm{nM}$ LDN193189 (Axon), $3 \mu \mathrm{M}$ CHIR99021 and $100 \mathrm{ng} / \mathrm{ml}$ hEGF in Advance DMEM F12 medium supplemented with 1X B27 supplement, 1X GlutaMax, 10 mM HEPES and 1X Pen-Strep. The differentiation medium was refreshed every 3 days for at least 40 days to achieve full colonic differentiation. The colon organoids were passaged and expanded every $10-14$ days at 1:6 density. To passage the organoids, the Matrigel domes containing the organoids were scrapped off the plate and resuspended in cold splitting media (Advance DMEM F12 medium supplemented with 1X GlutaMax, 10 mM HEPES and 1X Pen-Strep). The organoids were mechanically dislodged from the Matrigel dome and fragmented by pipetting in cold splitting media. The old Matrigel and splitting media were removed after pelleting cells and the organoids were resuspended in $100 \%$ Matrigel. $50 \mu \mathrm{L}$ Matrigel containing fragmentized colon organoids were plated in one well of a pre-warmed 24-well plate.

\section{Cell Lines}


HEK293T (human [Homo sapiens] fetal kidney) and Vero E6 (African green monkey [Chlorocebus aethiops] kidney) were obtained from ATCC (https://www.atcc.org/). Cells were cultured in Dulbecco's Modified Eagle Medium (DMEM) supplemented with $10 \%$ fetal bovine serum and 100 I.U./mL penicillin and $100 \mu \mathrm{g} / \mathrm{mL}$ streptomycin. All cell lines were incubated at $37^{\circ} \mathrm{C}$ with $5 \% \mathrm{CO} 2$.

\section{SARS-CoV-2 Pseudo-Entry Viruses}

Recombinant Indiana VSV (rVSV) expressing SARS-CoV-2 spikes were generated as previously described23. HEK293T cells were grown to $80 \%$ confluency before transfection with pCMV3SARS-CoV2-spike (kindly provided by Dr. Peihui Wang, Shandong University, China) using FuGENE 6 (Promega). Cells were cultured overnight at $37^{\circ} \mathrm{C}$ with $5 \% \mathrm{CO} 2$. The next day, medium was removed and VSV-G pseudotyped $\Delta$ G-luciferase (G* $\Delta G$-luciferase, Kerafast) was used to infect the cells in DMEM at an MOI of 3 for $1 \mathrm{hr}$ before washing the cells with 1X DPBS three times. DMEM supplemented with $2 \%$ fetal bovine serum and $100 \mathrm{I} . \mathrm{U} . / \mathrm{mL}$ penicillin and $100 \mu \mathrm{g} / \mathrm{mL}$ streptomycin was added to the infected cells and they were cultured overnight as described above. The next day, the supernatant was harvested and clarified by centrifugation at $300 \mathrm{~g}$ for $10 \mathrm{~min}$ and aliquots stored at $-80^{\circ} \mathrm{C}$.

\section{SARS-CoV-2 Viruses}

Severe acute respiratory syndrome coronavirus 2 (SARS-CoV-2), isolate USA-WA1/2020 (NR-52281) was deposited by the Center for Disease Control and Prevention and obtained through BEI Resources, NIAID, $\mathrm{NIH}$. SARS-CoV-2 was propagated in Vero E6 cells in DMEM supplemented with 2\% FBS, $4.5 \mathrm{~g} / \mathrm{L} \mathrm{D-}$ glucose, $4 \mathrm{mM}$ L-glutamine, $10 \mathrm{mM}$ Non-Essential Amino Acids, $1 \mathrm{mM}$ Sodium Pyruvate and $10 \mathrm{mM}$ HEPES. Infectious titers of SARS-CoV-2 were determined by plaque assay in Vero E6 cells in Minimum Essential Media supplemented with 2\% FBS, 4 mM L-glutamine, 0.2\% BSA, $10 \mathrm{mM}$ HEPES and 0.12\% $\mathrm{NaHCO} 3$ and $0.7 \%$ agar. All work involving live SARS-CoV-2 was performed in the CDC/USDA-approved BSL-3 facility of the Global Health and Emerging Pathogens Institute at the Icahn School of Medicine at Mount Sinai in accordance with institutional biosafety requirements.

\section{SARS-CoV-2 pseudo-entry virus infections}

To assay pseudo-typed virus infection on colon organoids, COs were seeded in 24 well plates. Pseudotyped virus was added at $\mathrm{MOI}=0.01$ plus polybrene at a final concentration of $8 \mu \mathrm{g} / \mathrm{mL}$, and the plate centrifuged for $1 \mathrm{hr}$ at $1200 \mathrm{~g}$. At $3 \mathrm{hpi}$, the infection medium was replaced with fresh medium. At $24 \mathrm{hpi}$, colon organoids were harvested for luciferase assays or immunostaining analysis. For chemical screening analysis, colon organoids were digested by TrypLE and seeded in 384 well plates at $1 \times 104$ cells per well. After chemical treatment, pseudo-typed virus was added at $\mathrm{MOI}=0.01$ and the plate centrifuged for $1 \mathrm{hr}$ at $1200 \mathrm{~g}$. At $24 \mathrm{hpi}$, hPSC-COs were harvested for luciferase assays according to the Luciferase Assay System protocol (Promega).

\section{SARS-CoV-2 virus infections}


hPSC-COs were infected with SARS-CoV-2 in the CO media at an MOI of 0.1, 0.05 or 0.01 as indicated and incubated at $37^{\circ} \mathrm{C}$. At $24 \mathrm{hpi}$, cells were washed three times with PBS and harvested for either RNA analysis or immunofluorescence staining.

Approximately $2.5 \times 105$ Vero E6 cells were pre-treated with the indicated compounds for $1 \mathrm{~h}$ prior to infection with SARS-CoV-2 at an MOI of 0.01 in DMEM supplemented with 2\% FBS, $4.5 \mathrm{~g} / \mathrm{L}$ D-glucose, 4 mM L-glutamine, 10 mM Non-Essential Amino Acids, 1 mM Sodium Pyruvate and 10 mM HEPES. At 24 hpi, cells were washed three times with PBS before harvesting for immunofluorescence staining or RNA or protein analysis.

Cells were lysed in RIPA buffer for protein analysis or fixed in 5\% formaldehyde for $24 \mathrm{~h}$ for immunofluorescent staining, prior to safe removal from the BSL-3 facility.

\section{Colon organoid processing and immunostaining}

The colon organoids were released from Matrigel using Cell Recovery Solution (Corning) on ice for $1 \mathrm{hr}$, followed by fixation in $4 \%$ paraformaldehyde for $4 \mathrm{hr}$ at $4^{\circ} \mathrm{C}$, washed twice with $1 \mathrm{X}$ PBS and allowed to sediment in $30 \%$ sucrose overnight. The organoids were then embedded in OCT (TissueTek) and cryosectioned at $10 \mu \mathrm{m}$ thickness. For indirect immunofluorescence staining, sections were rehydrated in $1 \mathrm{X}$ PBS for 5 min, permeabilized with $0.2 \%$ Triton in 1 X PBS for $10 \mathrm{~min}$, and blocked with blocking buffer containing $5 \%$ normal donkey serum in $1 \mathrm{X}$ PBS for $1 \mathrm{hr}$. The sections were then incubated with the corresponding primary antibodies diluted in blocking buffer at $4^{\circ} \mathrm{C}$ overnight. The following day, sections were washed three times with 1X PBS before incubating with fluorophore-conjugated secondary antibody for one hr at RT. The sections were washed three times with 1X PBS and mounted with Prolong Gold Antifade mounting media with DAPI (Life technologies). Images were acquired using an LSM880 Laser Scanning Confocal Microscope (Zeiss) and processed with Zen or Imaris (Bitplane) software.

\section{Immunofluorescent staining}

Organoids and tissues were fixed in 4\% PFA for 20 min at RT, blocked in Mg2+/Ca2+ free PBS plus 5\% horse serum and $0.3 \%$ Triton-X for $1 \mathrm{hr}$ at RT, and then incubated with primary antibody at $4^{\circ} \mathrm{C}$ overnight. The information for primary antibodies is provided in Extended Data Table 2. Secondary antibodies included donkey anti-mouse, goat, rabbit or chicken antibodies conjugated with Alexa-Fluor-488, AlexaFluor-594 or Alexa-Fluor- 647 fluorophores (1:500, Life Technologies). Nuclei were counterstained by DAPI.

\section{Western blot}

Protein was extracted from cells in Radioimmunoprecipitation assay (RIPA) lysis buffer containing $1 \mathrm{X}$ Complete Protease Inhibitor Cocktail (Roche) and 1X Phenylmethylsulfonyl fluoride (Sigma Aldrich) prior to safe removal from the BSL-3 facility. Samples were analysed by SDS-PAGE and transferred onto nitrocellulose membranes. Proteins were detected using rabbit polyclonal anti-GAPDH (Sigma Aldrich, G9545), mouse monoclonal anti-SARS-CoV-2 Nucleocapsid [1C7] and mouse monoclonal anti-SARS-CoV- 
2 Spike [2B3E5] protein (a kind gift by Dr. T. Moran, Center for Therapeutic Antibody Discovery at the Icahn School of Medicine at Mount Sinai). Primary antibodies were detected using Fluorophoreconjugated secondary goat anti-mouse (IRDye 680RD, 926-68070) and goat anti-rabbit (IRDye 800CW, 926-32211) antibodies. Antibody-mediated fluorescence was detected on a LI-COR Odyssey CLx imaging system and analyzed using Image Studio software (LI-COR).

\section{Single cell organoid preparation for scRNA-sequencing}

The colon organoids cultured in Matrigel domes were dissociated into single cells using $0.25 \%$ Trypsin (Gibco) at $37^{\circ} \mathrm{C}$ for $10 \mathrm{~min}$, and the trypsin was then neutralized with DMEM F12 supplemented with $10 \%$ FBS. The dissociated organoids were pelleted and resuspended with L15 Medium (Gibco) supplemented with $10 \mathrm{mM}$ HEPES, and $10 \mathrm{ng} / \mathrm{ml}$ DNasel (Sigma). The resuspended organoids were then placed through a $40 \mu \mathrm{m}$ filter to obtain a single cell suspension, and stained with DAPI followed by sorting of live cells using an ARIA II flow cytometer (BD Biosciences). The live colonic single cell suspension was transferred to the Genomics Resources Core Facility at Weill Cornell Medicine to proceed with the Chromium Single Cell 3' Reagent Kit v3 (10x Genomics, product code \# 1000075) using 10X Genomics Chromium Controller. A total of 10,000 cells were loaded into each channel of the Single-Cell A Chip to target 8000 cells. Briefly, according to manufacturer's instruction, the sorted cells were washed with $1 \times$ PBS $+0.04 \%$ BSA, counted by a Bio-Rad TC20 Cell Counter, and cell viability was assessed and visualized. A total of 10,000 cells and Master Mixes were loaded into each channel of the cartridge to generate the droplets on Chromium Controller. Beads-in-Emulsion (GEMs) were transferred and GEMs-RT was undertaken in droplets by PCR incubation. GEMs were then broken and pooled fractions recovered. After purification of the first-strand CDNA from the post GEM-RT reaction mixture, barcoded, full-length CDNA was amplified via PCR to generate sufficient mass for library construction. Enzymatic fragmentation and size selection were used to optimize the cDNA amplicon size. TruSeq Read 1 (read 1 primer sequence) was added to the molecules during GEM incubation. P5, P7, a sample index, and TruSeq Read 2 (read 2 primer sequence) were added via End Repair, A-tailing, Adaptor Ligation, and PCR. The final libraries were assessed by Agilent Technology 2100 Bioanalyzer and sequenced on Illumina NovaSeq sequencer with pair-end 100 cycle kit $(28+8+91)$.

\section{Sequencing and gene expression UMI counts matrix generation}

T FASTQ files were imported to a 10x Cell Ranger - data analysis pipeline (v3.0.2) to align reads, generate feature-barcode matrices and perform clustering and gene expression analysis. In a first step, cellranger mkfastq demultiplexed samples and generated fastq files; and in the second step, cellranger count aligned fastq files to the reference genome and extracted gene expression UMI counts matrix. In order to measure viral gene expression, we built a custom reference genome by integrating the four virus genes and luciferase into the $10 \mathrm{X}$ pre-built human reference (GRCh38 v3.0.0) using cellranger mkref. The sequences of four viral genes (VSV-N VSV-NS, VSV-M and VSV-L) were retrieved from NCBI (https://www.ncbi.nlm.nih.gov/nuccore/33587그), and the sequence of the luciferase was retrieved from HIV-Luc. 


\section{Single-cell RNA-seq data analysis}

We filtered cells with less than 300 or more than 8000 genes detected as well as cells with mitochondria gene content greater than $30 \%$, and used the remaining cells ( 6175 cells for the uninfected sample and 2962 cells for the infected sample) for downstream analysis. We normalized the gene expression UMI counts for each sample separately using a deconvolution strategy 24 implemented by the R scran package (v.1.14.1). In particular, we pre-clustered cells in each sample using the quickClusterfunction; we computed size factor per cell within each cluster and rescaled the size factors by normalization between clusters using the computeSumFactors function; and we normalized the UMI counts per cell by the size factors and took a logarithm transform using the normalize function. We further normalized the UMI counts across samples using the multiBatchNorm function in the R batchelor package (v1.2.1). We identified highly variable genes using the FindVariableFeatures function in the R Seurat (v3.1.0)25, and selected the top 3000 variable genes after excluding mitochondria genes, ribosomal genes and dissociation-related genes. The list of dissociation-related genes was originally built on mouse data26, we converted them to human ortholog genes using Ensembl BioMart. We aligned the two samples based on their mutual nearest neighbors (MNNs) using the fastMNN function in the R batchelor package, this was done by performing a principal component analysis (PCA) on the highly variable genes and then correcting the principal components (PCs) according to their MNNs. We selected the corrected top 50 PCs for downstream visualization and clustering analysis. We ran the uniform manifold approximation and projection (UMAP) dimensional reduction using the RunUMAP function in the R Seurat25 package with training epochs setting to 2000 . We clustered cells into eight clusters by constructing a shared nearest neighbor graph and then grouping cells of similar transcriptome profiles using the FindNeighbors function and FindClusters function (resolution set to 0.2 ) in the R Seurat package. We identified marker genes for each cluster by performing differential expression analysis between cells inside and outside that cluster using the FindMarkers function in the R Seurat package. After reviewing the clusters, we merged four clusters that were likely from stem cell population into a single cluster ( $L G R 5+$ or $B M I 1+$ stem cells) and kept the other four clusters (KRT20+epithelial cells, MUC2+ goblet cells, EPHB2+TA cells, and $C H G A+$ NE cells) for further analysis. We re-identified marker genes for the merged five clusters and selected the top 10 positive marker genes per cluster for heatmap plot using the DoHeatmap function in the R Seurat package25.

\section{In vivo transplantation and drug evaluation}

hPSC-COs were harvested by cell scraper, mixed with $20 \mu \mathrm{l}$ Matrigel (Corning) and transplanted under the kidney capsule of 7-9 weeks old male NSG mice. Two weeks post- transplantation, SARS-CoV-2 pseudoentry virus was inoculated locally at 1x103 FFU. At $24 \mathrm{hpi}$, the mice were euthanized and used for immunohistochemistry analysis.

To determine the MPA's activity in vivo, the mice were treated with $50 \mathrm{mg} / \mathrm{kg}$ MPA in (10\%DMSO/90\% corn oil) by IP injection. Two hours after drug administration, SARS- CoV-2 pseudo-entry virus was 
inoculated locally at 1x103 FFU. At $24 \mathrm{hpi}$, the mice were euthanized and used for immunohistochemistry analysis.

All animal work was conducted in agreement with NIH guidelines and approved by the WCM Institutional Animal Care and Use Committee (IACUC) and the Institutional Biosafety Committee (IBC).

\section{High throughput chemical screening}

To perform the high throughput small molecule screen, hPSC-COs were dissociated using TrypLE for 20 min in a $37^{\circ} \mathrm{C}$ waterbath and replated into $10 \%$ Matrigel-coated 384 - well plates at 20,000 cells/40 $\mu \mathrm{l}$ medium/well. After $6 \mathrm{hr}$, cells were treated with compounds from an in-house library of 1280 FDAapproved drugs (Prestwick) at $10 \mu \mathrm{M}$. DMSO treatment was used as a negative control. One hour late, cells will be infected with SARS-CoV-2 pseudo virus (MOI=0.01). After $24 \mathrm{hpi}$, hPSC-COs were harvested for luciferase assay following the Luciferase Assay System protocol (Promega).

\section{RNA-Seq following viral infections}

Organoid infections were performed at an $\mathrm{MOI}$ of 0.1 and harvested at $24 \mathrm{hpi}$ in DMEM supplemented with $0.3 \%$ BSA, $4.5 \mathrm{~g} / \mathrm{L}$ D-glucose, $4 \mathrm{mM}$ L-glutamine and $1 \mu \mathrm{g} / \mathrm{ml}$ TPCK- trypsin. Total RNA was extracted in TRIzol (Invitrogen) and DNase I treated using Direct- zol RNA Miniprep kit (Zymo Research) according to the manufacturer's instructions. RNA- seq libraries of polyadenylated RNA were prepared using the TruSeq RNA Library Prep Kit v2 (Illumina) or TruSeq Stranded mRNA Library Prep Kit (Illumina) according to the manufacturer's instructions. cDNA libraries were sequenced using an Illumina NextSeq 500 platform. Raw reads were aligned to the human genome (hg19) using the RNA-Seq Aligment App on Basespace (Illumina, CA), following differential expression analysis using DESeq227. Differentially expressed genes (DEGs) were characterized for each sample ( $p$ adjusted-value $<0.05$ ). Volcano plots were constructed using custom scripts in R.

\section{Statistical analysis}

$\mathrm{N}=3$ independent biological replicates were used for all experiments unless otherwise indicated. n.s. indicates non-significance. $P$-values were calculated by unpaired two-tailed Student's t-test unless otherwise indicated. ${ }^{*} p<0.05,{ }^{*} p<0.01$ and ${ }^{* *} p<0.001$.

\section{Declarations}

\section{Acknowledgements}

This work was supported by the Department of Surgery, Weill Cornell Medicine (T.E., F.P, S.C.), by the Defense Advanced Research Projects Agency (DARPA) through a contract with B.T. (DARPA-16-35INTERCEPT-FP-006) and by the Jack Ma Foundation to D.D.H. The authors would like to thank Dr. Tom Moran, Center for Therapeutic Antibody Discovery at the Icahn School of Medicine at Mount Sinai for 
providing anti-SARS-Cov- SPIKE antibody. We are also very grateful for technical support and advice provided by Lee Cohen-Gould and Robert Lance Furler in the Cell Screening Core Facility of WCM.

\section{Author contributions}

S. C., T. E., F. P., D. H., R.S, H. J. C., H. W., conceived and designed the experiments.

X. D., Y.H., L. Y., F. P., performed organoid differentiation, in vivo transplantation, pseudo-virus infection and drug screening.

P. W, Y. Hu., performed SARS2-CoV-2 pseudo-entry virus related experiments.

B. N., and B. T., performed SARS2-CoV-2 related experiments.

T. Z., J. X. Z., D. X., X. W., performed the scRNA-sequencing and bioinformatics analyses.

\section{Data Availability}

scRNA-seq and RNA-seq data is available from the GEO repository database, accession number GSE147975.

\section{References}

1. https://journals.Iww.com/ajg/Documents/COVID_Digestive_Symptoms_AJG_pdf.

2. Crespo, M. et al. Colonic organoids derived from human induced pluripotent stem cells for modeling colorectal cancer and drug testing. Nat Med 23, 878-884, doi:10.1038/nm.4355 (2017).

3. Munera, J. O. et al. Differentiation of Human Pluripotent Stem Cells into Colonic Organoids via Transient Activation of BMP Cell Stem Cell 21, 51-64 e56, doi:10.1016/j.stem.2017.05.020 (2017).

4. Hoffmann, et al. SARS-CoV-2 Cell Entry Depends on ACE2 and TMPRSS2 and Is Blocked by a Clinically Proven Protease Inhibitor. Cell, doi:10.1016/j.cell.2020.02.052 (2020).

5. Whitt, M. A. Generation of VSV pseudotypes using recombinant DeltaG-VSV for studies on virus entry, identification of entry inhibitors, and immune responses to vaccines. $J$ Virol Methods 169, 365-374, doi:10.1016/j.jviromet.2010.08.006 (2010).

6. Nie, et al. Establishment and validation of a pseudovirus neutralization assay for SARS-CoV-2. Emerg Microbes Infect 9, 680-686, doi:10.1080/22221751.2020.1743767 (2020).

7. https://www.fda.gov/news-events/press-announcements/coronavirus-covid-19- update-dailyroundup-march-30-2020.

8. https://doi.org/10.1101/2020.03.24.004655.

9. Law, H. K. et al. Chemokine up-regulation in SARS-coronavirus-infected, monocyte-derived human dendritic cells. Blood 106, 2366-2374, doi:10.1182/blood-2004-10-4166 (2005). 
10. Channappanavar, R. et al. Dysregulated Type I Interferon and Inflammatory Monocyte-Macrophage Responses Cause Lethal Pneumonia in SARS-CoV-Infected Mice. Cell Host Microbe 19, 181-193, doi:10.1016/j.chom.2016.01.007 (2016).

11. Zhou, P. et al. A pneumonia outbreak associated with a new coronavirus of probable bat Nature $\mathbf{5 7 9}$, 270-273, doi:10.1038/s41586-020-2012-7 (2020).

12. Ou, X. et al. Characterization of spike glycoprotein of SARS-CoV-2 on virus entry and its immune cross-reactivity with SARS-CoV. Nat Commun 11, 1620, doi:10.1038/s41467-020-15562-9 (2020).

13. Kuypers, D. R. et al. Consensus report on therapeutic drug monitoring of mycophenolic acid in solid organ transplantation. Clin J Am Soc Nephro/ 5, 341- 358, doi:10.2215/CJN.07111009 (2010).

14. Franklin, T. J. \& Cook, J. M. The inhibition of nucleic acid synthesis by mycophenolic acid. Biochem J 113, 515-524, doi:10.1042/bj1130515 (1969).

15. Chapuis, A. G. et al. Effects of mycophenolic acid on human immunodeficiency virus infection in vitro and in Nat Med 6, 762-768, doi:10.1038/77489 (2000).

16. Diamond, M. S., Zachariah, M. \& Harris, E. Mycophenolic acid inhibits dengue virus infection by preventing replication of viral RNA. Virology 304, 211-221, doi:10.1006/viro.2002.1685 (2002).

17. Cheng, K. W. et al. Thiopurine analogs and mycophenolic acid synergistically inhibit the papain-like protease of Middle East respiratory syndrome coronavirus. Antiviral Res 115, 9-16, doi:10.1016/j.antiviral.2014.12.011 (2015).

18. Lin, M. H. et al. Disulfiram can inhibit MERS and SARS coronavirus papain-like proteases via different modes. Antiviral Res 150, 155-163, doi:10.1016/j.antiviral.2017.12.015 (2018).

19. https://www.biorxiv.org/content/10.1101/2020.03.22.002386v2.

20. Al Ghamdi, M. et al. Treatment outcomes for patients with Middle Eastern Respiratory Syndrome Coronavirus (MERS CoV) infection at a coronavirus referral center in the Kingdom of Saudi Arabia. BMC Infect Dis 16, 174, doi:10.1186/s12879-016-1492-4 (2016).

21. https://www.drugs.com/mmx/quinacrine-hydrochloride.html.

22. Lane, T. R., Comer, J. E., Freiberg, A. N., Madrid, P. B. \& Ekins, S. Repurposing Quinacrine against Ebola Virus Infection In Antimicrob Agents Chemother 63, doi:10.1128/AAC.01142-19 (2019).

23. Zhao, X. et al. Immunization-Elicited Broadly Protective Antibody Reveals Ebolavirus Fusion Loop as a Site of Vulnerability. Cell 169, 891-904 e815, doi:10.1016/j.cell.2017.04.038 (2017).

24. Lun, A. T., Bach, K. \& Marioni, J. C. Pooling across cells to normalize single-cell RNA sequencing data with many zero counts. Genome Biol 17, 75, doi:10.1186/s13059-016-0947-7 (2016).

25. Stuart, T. et al. Comprehensive Integration of Single-Cell Data. Cell 177, 1888- 1902 e1821, doi:10.1016/j.cell.2019.05.031 (2019).

26. van den Brink, S. C. et al. Single-cell sequencing reveals dissociation-induced gene expression in tissue subpopulations. Nat Methods 14, 935-936, doi:10.1038/nmeth.4437 (2017).

27. Love, M. I., Huber, W. \& Anders, S. Moderated estimation of fold change and dispersion for RNA-seq data with DESeq2. Genome Bio/ 15, 550, doi:10.1186/s13059-014-0550-8 (2014). 
Figure 1.

a.

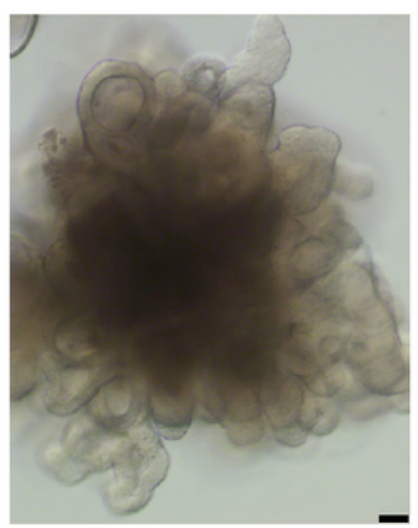

b.
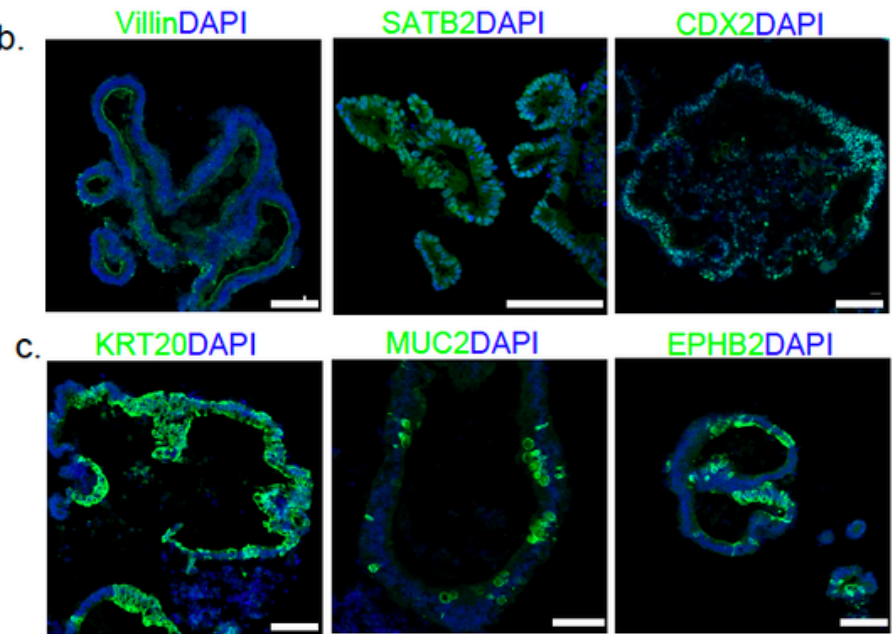

MUC2DAPI

EPHB2DAPI

CHGADAPI
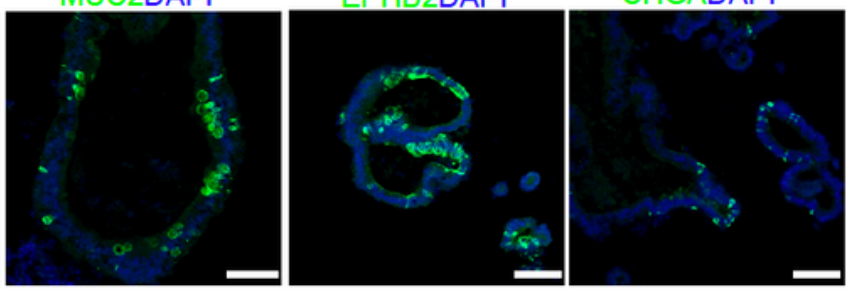

d.
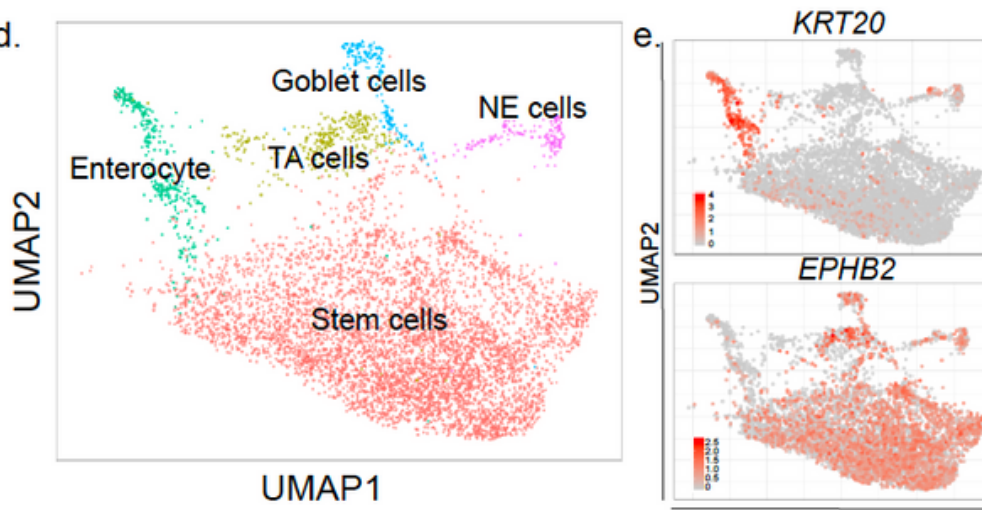

MUC2

CHGA

UMAP1
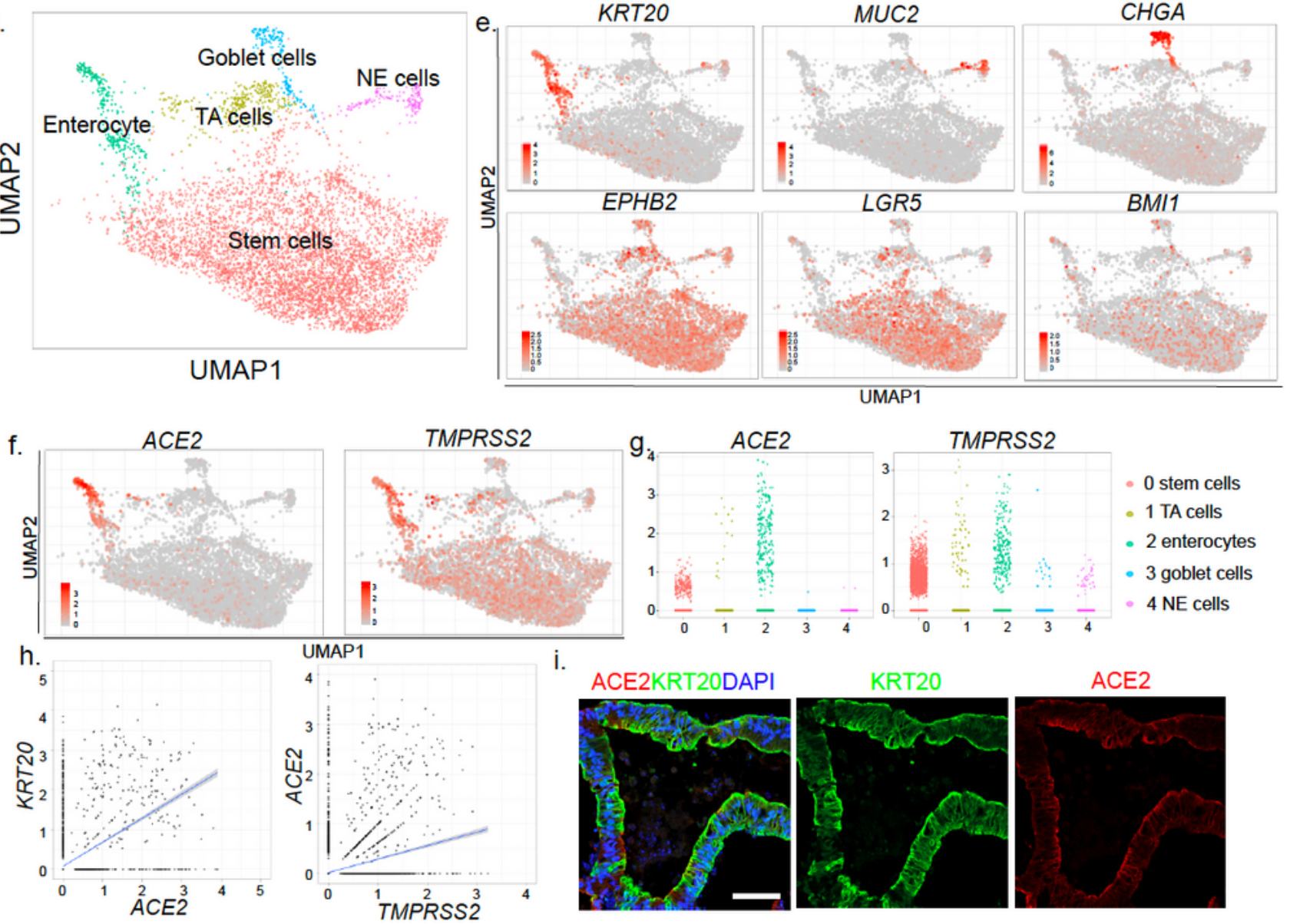

TMPRSS2

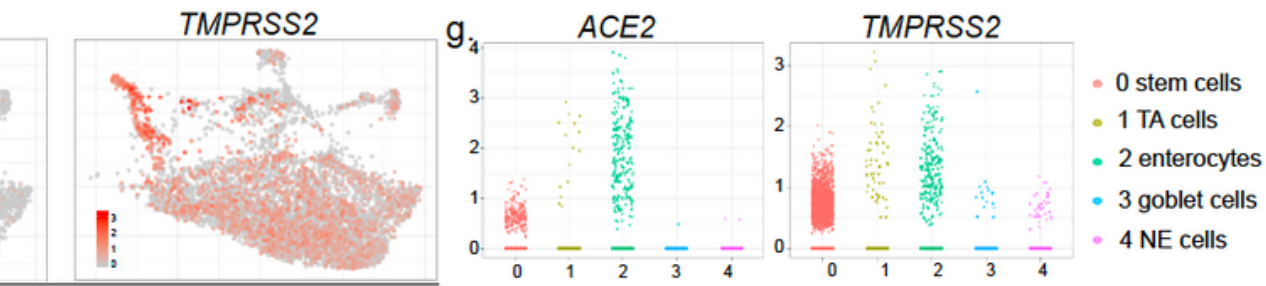

TMPRSS2
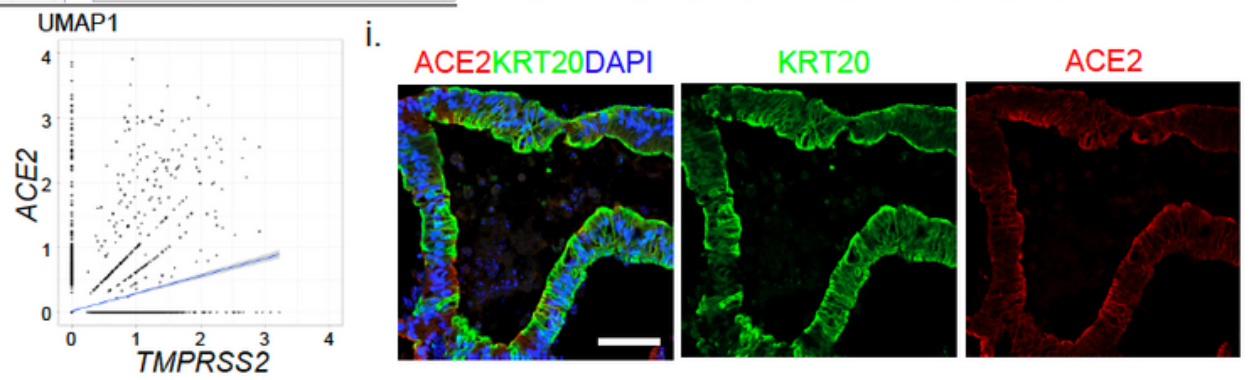

\section{Figure 1}

ACE2 and TMPRSS2 are expressed in hPSC-COs. a, Phase contrast image of a representative hPSCderived colon organoid (CO). Scale bar $=100 \mu \mathrm{m}$. b, c, Confocal imaging of hPSC-COs stained with antibodies against (b) markers for colon cell fate, including Villin, SATB2, CDX2, or (c) KRT20, MUC2, 
EPHB2, and CHGA; DAPI stains nuclei. Scale bar $=100 \mu \mathrm{m}$. d, UMAP of hPSC-CO cell types. e, UMAP of markers for specific colonic cell fates markers, including KRT20, MUC2, CHGA, EPHB2, LGR5, and BMI1. f, UMAP of ACE2 and TMPRSS2. g, Jitter plots for expression levels of ACE2 and TMPRSS2. h, 2D correlation of expression levels for KRT20 and ACE2; ACE2 and TMPRSS2. i, Representative confocal images of hPSC-COs co-stained with antibodies recognizing ACE2 and KRT20. DAPI stains nuclei. Scale bar $=100 \mu \mathrm{m}$.

Figure 2.

a.

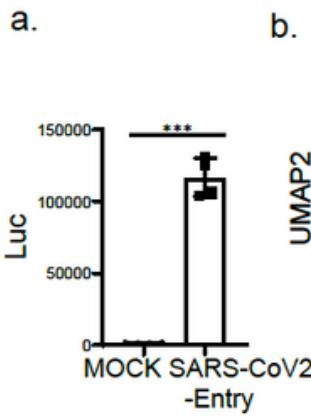

b.

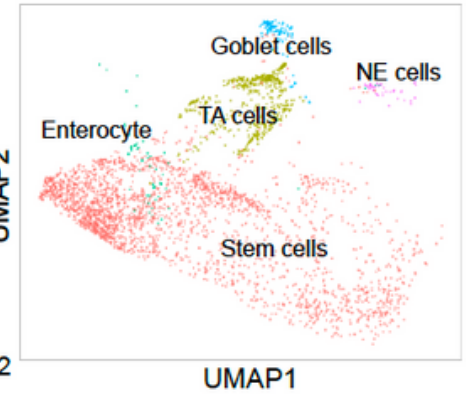

c.

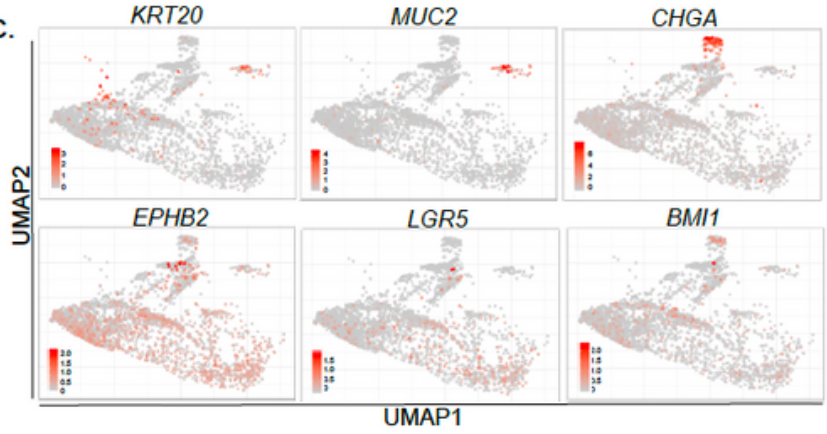

d.

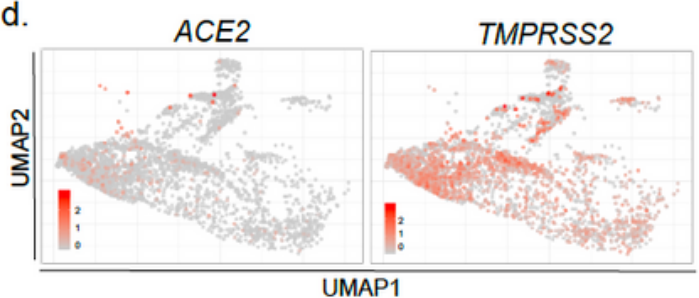

e.

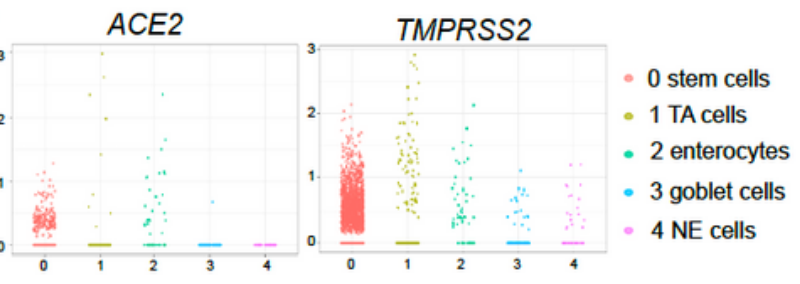

f.

VSV-N

VSV-NS

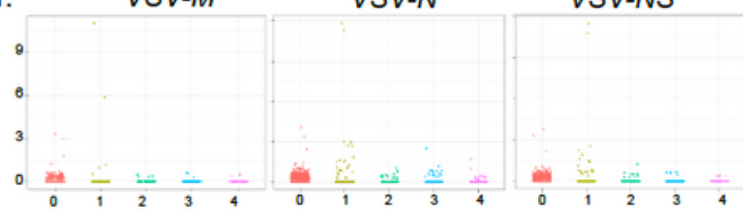

h.
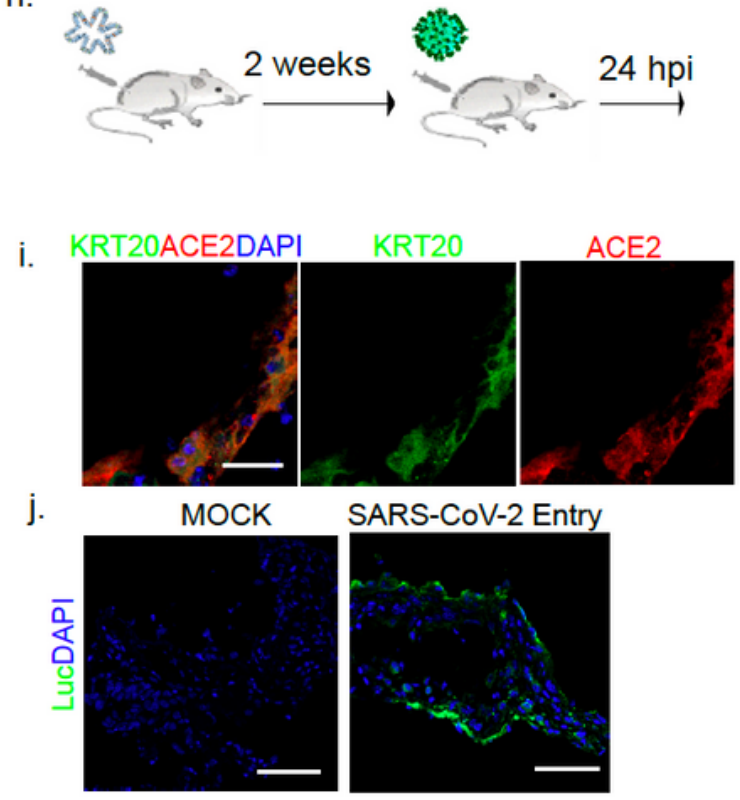

g. VillinLucDAP|

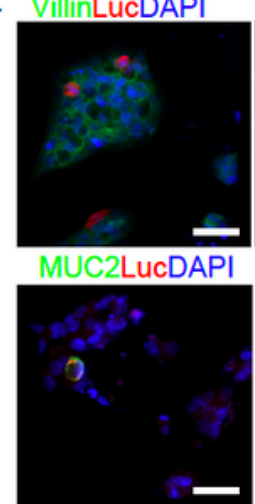

CDX2LucDAPI KRT20LucDAPI
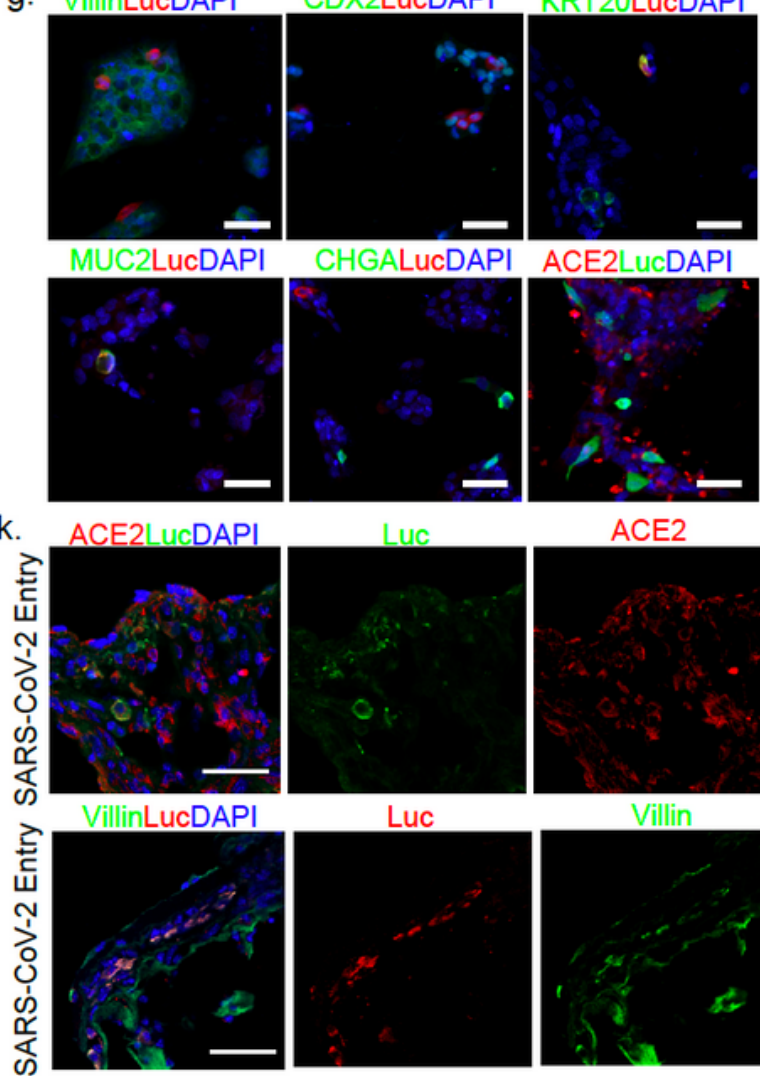

CHGALucDAPI ACE2LucDAPI
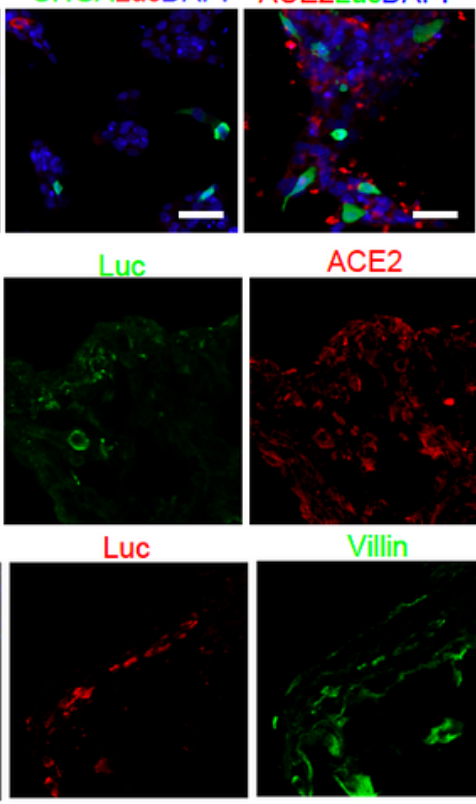

ACE2

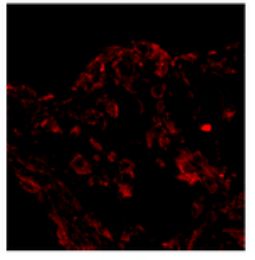

Vilin

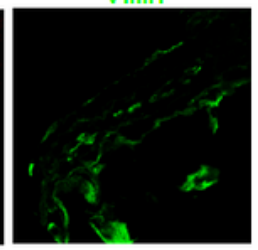


hPSC-COs can be infected by a SARS-CoV-2 pseudo-entry virus both in vitro and in vivo. a, Luciferase activity in lysates from hPSC-derived colonic cells at 24 hpi following exposure to SARS-CoV-2 pseudoentry virus at MOI=0.01. b, UMAP of hPSC-COs at 24 hpi with SARS-CoV-2 pseudo-entry virus. c, UMAP of markers for specific colonic cell fates, including KRT20, MUC2, CHGA, EPHB2, LGR5, and BMI1. d, UMAP of ACE2 and TMPRSS2. e, Jitter plots of ACE2 and TMPRSS2 transcript levels. f, Jitter plots of VSV-M, VSV-N and VSV-NS transcript levels. g, Immunocytochemistry staining of hPSC-CO cells infected with SARS-CoV-2 pseudo-entry virus (MOI=0.01) at $24 \mathrm{hpi}$ using antibodies against luciferase and specific markers for colonic cell fate, including Villin, CDX2, KRT20, MUC2, CHGA, and ACE2. Scale bar $=50 \mu \mathrm{m}$. h, Schematic for the in vivo infection. i, Representative confocal image of a hPSC-CO xenograft at $24 \mathrm{hpi}$ stained with antibodies against ACE2 and KRT20. DAPI stains nuclei. Scale bar $=25 \mu \mathrm{m}$. j, Representative confocal image of a hPSC-CO xenograft at $24 \mathrm{hpi} \mathrm{(1X103} \mathrm{FFU)} \mathrm{stained} \mathrm{with} \mathrm{antibody} \mathrm{against} \mathrm{luciferase.}$ DAPI stains nuclei. Scale bar $=75 \mu \mathrm{m}$. k, Representative confocal images of hPSC-CO xenografts at 24 hpi stained with antibodies against luciferase, ACE2 and Villin. DAPI stains nuclei. Scale bar $=50 \mu \mathrm{m}$. Data in (a) is presented as mean \pm STDEV. $N=3$. $P$ values were calculated by unpaired two-tailed Student's t-test. ***P $<0.001$. 
Figure 3 .
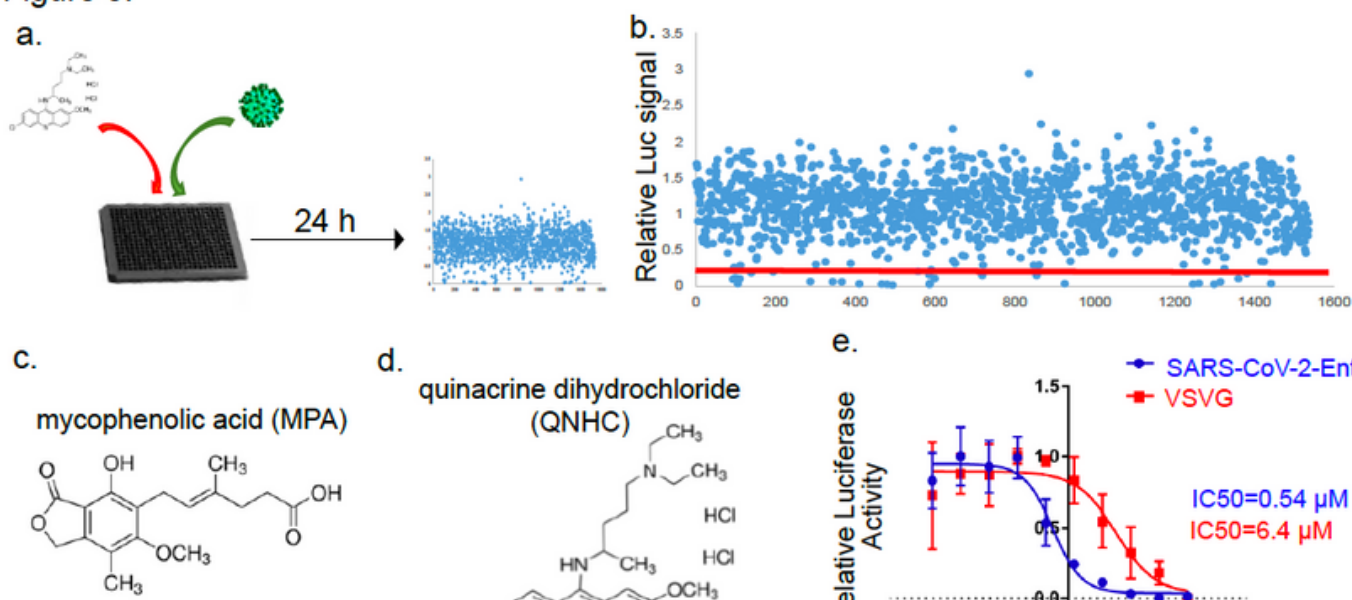

d. quinacrine dihydrochloride
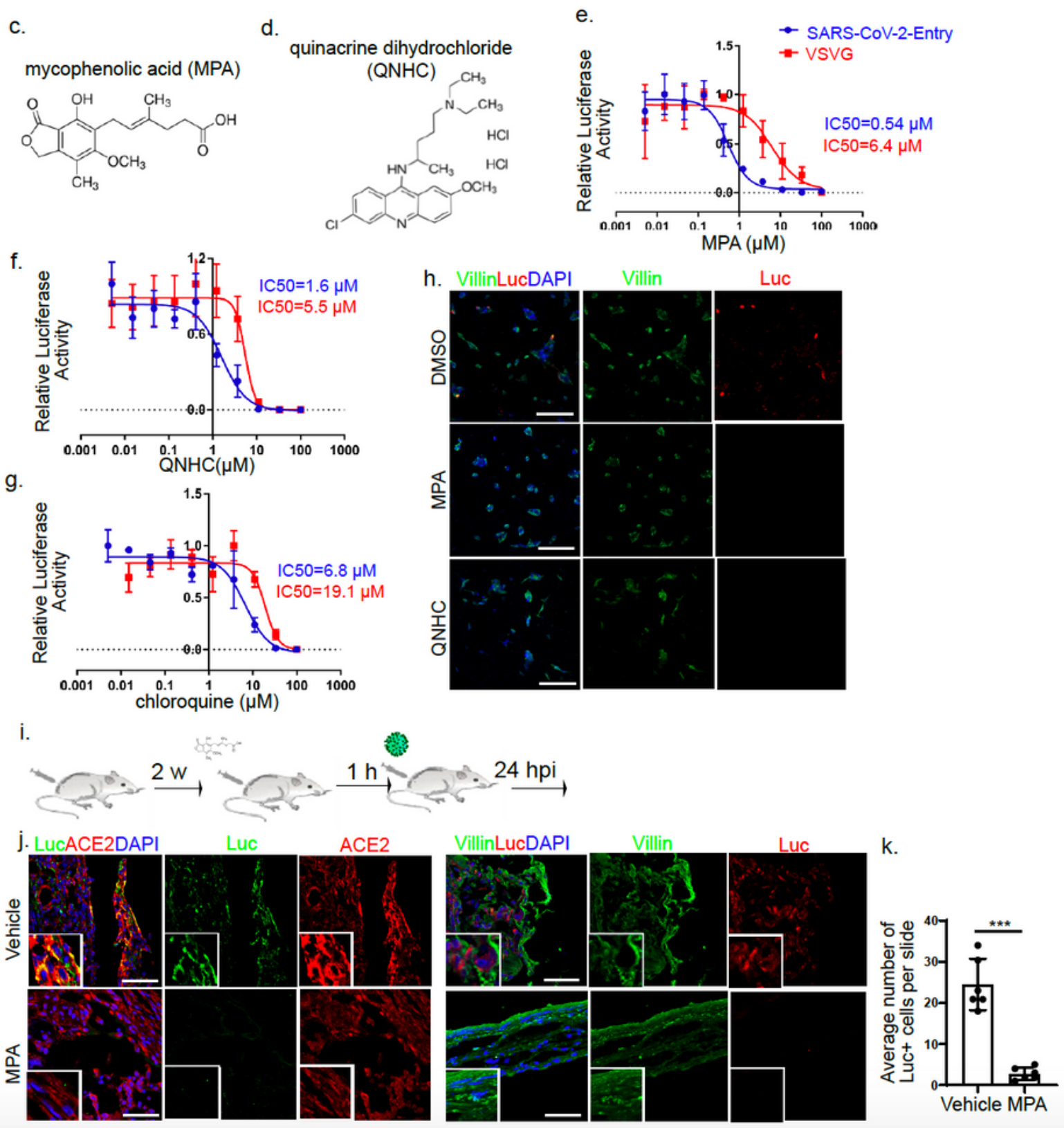

\section{Figure 3}

A high throughput screen to identify drugs that block SARS-CoV-2 entry. a, Schematic describing the high throughput drug screen platform. b, Primary screen data. c, d, Chemical structure of mycophenolic acid (MPA, c) and quinacrine dihydrochloride (QNHC, d). e-g, Inhibition curves for MPA (e), QNHC (f) and chloroquine (g) comparing hPSC-COs infected with SARS-CoV-2 pseudo-entry or control VSVG viruses (for both, MOI= 0.01). h, Immunofluorescent staining for luciferase in $3 \mu \mathrm{M}$ MPA, $4.5 \mu \mathrm{M}$ QNHC or DMSO- 
treated $\mathrm{hPSC}-\mathrm{COs}$ at $24 \mathrm{hpi}(\mathrm{MOI}=0.01)$. Scale bar $=100 \mu \mathrm{m}$. i. Scheme of the in vivo drug evaluation. $\mathrm{j}$. Representative confocal images of MPA or DMSO-treated hPSC-CO xenografts at $24 \mathrm{hpi}$ stained with antibodies against luciferase, ACE2 and Villin. DAPI stains nuclei. Scale bar $=50 \mu \mathrm{m}$. k. Quantification of the average number of Luc+ cells per xenograft. ( $N=6$ xenografts, 5 slides/xenografts).

Figure 4.
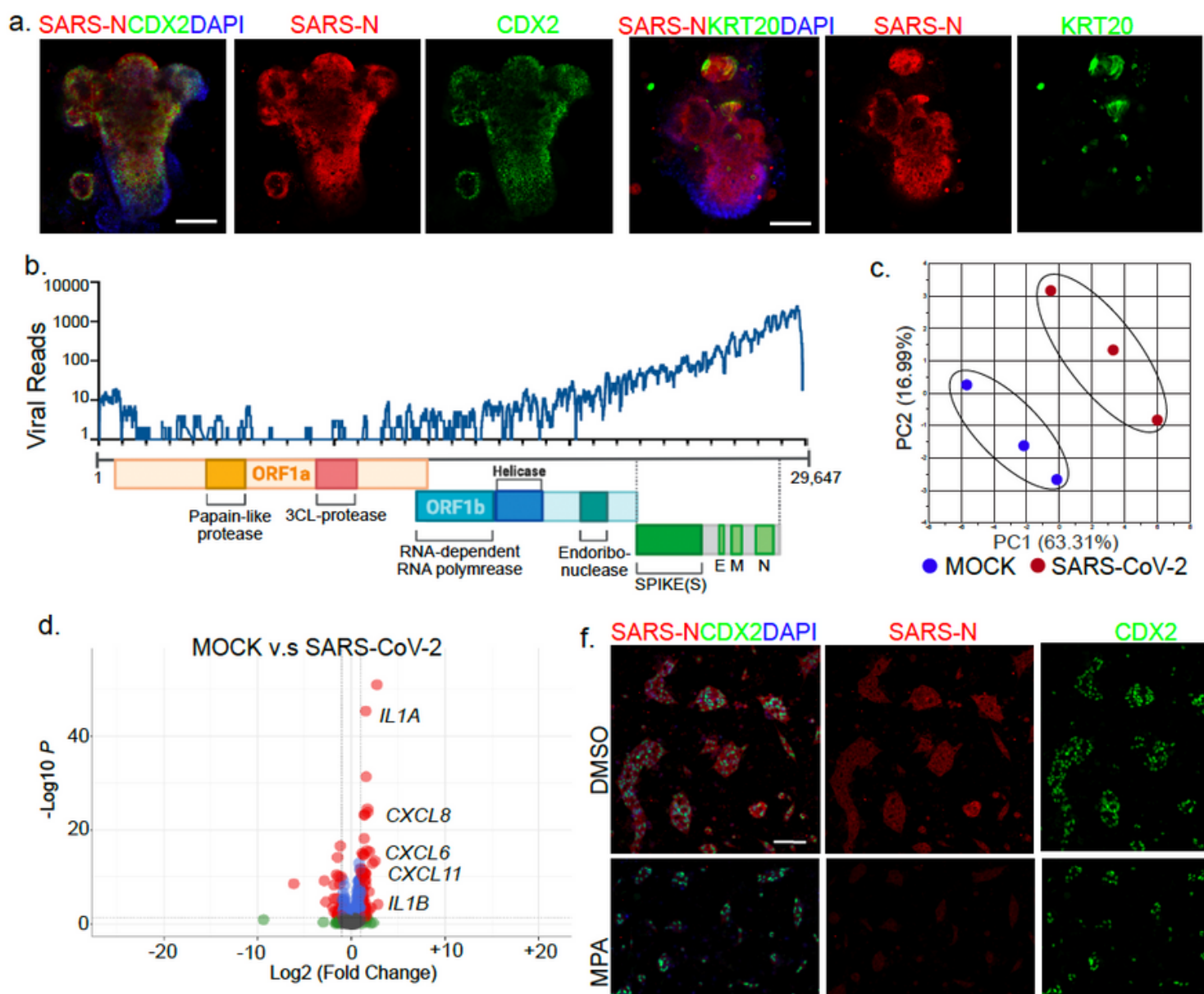

e.
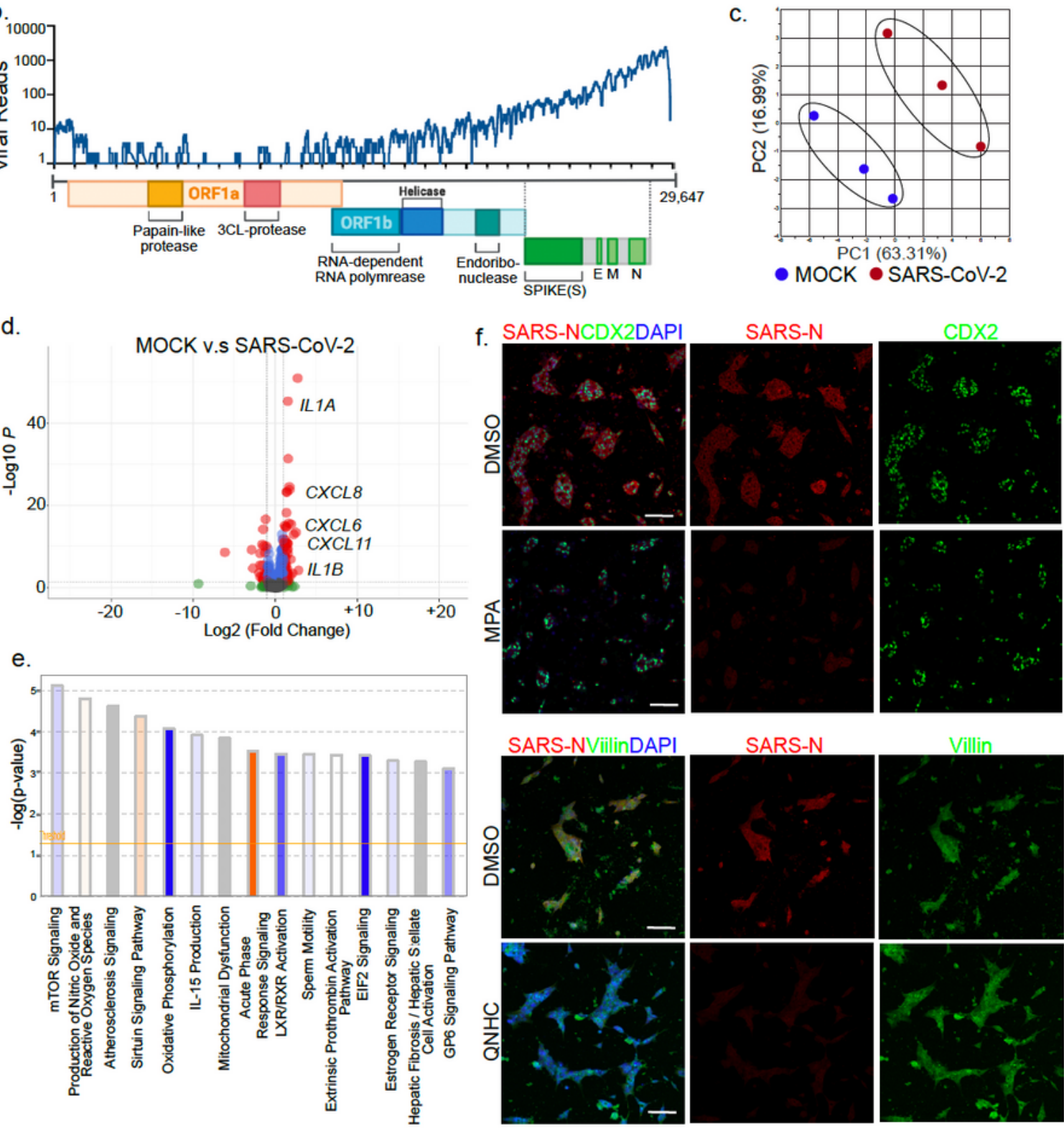

SARS-N
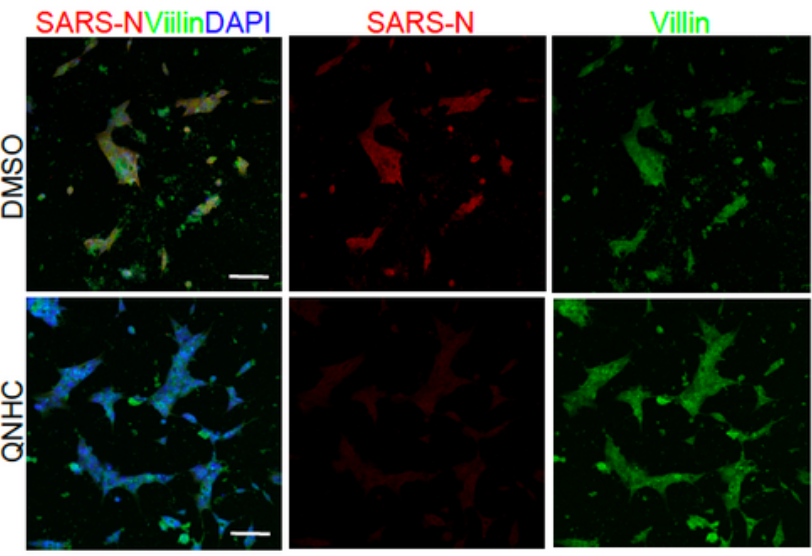

Figure 4 
Quinacrine dihydrochloride and mycophenolic acid block the infection of SARS-CoV-2 virus. a, Immunofluorescent staining to detect SARS-CoV-2-nucleocapsid protein (SARS-N) in COs co-stained for CDX2 or KRT20. DAPI stains nuclei. Scale bar $=100 \mu \mathrm{m}$. b. Alignment of the transcriptome with the viral genome in SARS-CoV-2 infected hPSC-COs. Schematic noted of the SARS-CoV-2 genome. c, PCA plot of gene expression profiles from MOCK-infected and SARS-CoV-2 infected hPSC-COs at $24 \mathrm{hpi}(\mathrm{MOI}=0.1)$. d, Volcano plots indicating differentially expressed genes in hPSC-COs comparing MOCK and SARS-CoV-2 infected hPSC-COs at $24 \mathrm{hpi}(\mathrm{MOl}=0.1)$. Differentially expressed genes ( $\mathrm{p}$-adjusted value $<0.05)$ with a log2 (Fold Change) $>2$ are indicated in red. Non-significant differentially expressed genes with a log2 (Fold Change) $>2$ are indicated in green. e, IPA of differentially expressed genes in d. $f$, Immunofluorescent staining of SARS-CoV-2 nucleocapsid protein (SARS-N) of $3 \mu \mathrm{M}$ MPA, $4.5 \mu \mathrm{M}$ QNHC or DMSO-treated hPSC-COs at 24 hour post-SARS-CoV-2 infection (MOI=0.1). Scale bar $=100 \mu \mathrm{m}$.

\section{Supplementary Files}

This is a list of supplementary files associated with this preprint. Click to download.

- ExtendeddataTables.pdf

- ExtendeddataFigures.pdf 